Z Gerontol Geriat 2023 · 56:125-131 https://doi.org/10.1007/s00391-022-02023-9

Eingegangen: 5. November 2021

Angenommen: 10. Januar 2022

Online publiziert: 4. Februar 2022

(c) Der/die Autor(en) 2022

\section{Balance Evaluation Systems Test: deutsche Übersetzung, kulturelle Anpassung und erste Ergebnisse zu Gütekriterien}

\author{
Maren Haselwander' $\cdot$ Yannick Henes ${ }^{1} \cdot$ Matthias Weisbrod $^{2,3} \cdot$ Gudrun Diermayr $^{1}$ \\ ${ }^{1}$ Fakultät für Therapiewissenschaften, SRH Hochschule Heidelberg, Heidelberg, Deutschland \\ ${ }^{2}$ Abteilung für Allgemeine Psychiatrie, Zentrum für Psychosoziale Medizin, Universitätsklinikum \\ Heidelberg, Heidelberg, Deutschland \\ ${ }^{3}$ Abteilung für Psychiatrie und Psychotherapie, SRH Klinikum Karlsbad - Langensteinbach, Karlsbad, \\ Deutschland
}

Hintergrund: Der Balance Evaluation Systems Test (BESTest) evaluiert die Balancefähigkeit und identifiziert die dem Balancedefizit zugrunde liegenden Systeme. Ziel: Primäre Ziele waren die Übersetzung und kulturelle Anpassung des BESTest ins Deutsche. Sekundäres Ziel war die Testung der Gütekriterien. Methode: Der Übersetzungs- und Anpassungsprozess erfolgte in 7 Schritten in Anlehnung an internationale Richtlinien. Zur Testung der Gütekriterien wurden Personen mit subjektivem oder objektivem Balancedefizit eingeschlossen. Die Testung beinhaltete die Bestimmung der internen Konsistenz mittels Cronbachs a und der Kriteriumsvalidität mittels Korrelation mit der Berg Balance Scale (BBS). Die Konstruktvalidität wurde mit der Activities-Specific Balance Confidence-Scale (ABC-D Scale) sowie anhand der Methode der bekannten Gruppen (gestürzte vs. nichtgestürzte Teilnehmende) untersucht.

Ergebnisse: Von 27 Personen liegen Ergebnisse zur Testung der Gütekriterien vor. Cronbachs a beträgt 0,95 für die Gesamtskala. Der Zusammenhang der BESTest-Werte mit denen der BBS lag mit Spearmans rho bei $\rho=0,84(p<0,001)$ und mit denen der ABC-D Scale bei $\rho=0,61(p<0,001)$. Gestürzte und Nichtgestürzte zeigten nur im Trend unterschiedliche BESTest Werte $(p=0,057)$.

Diskussion: Mit dieser Arbeit steht eine von der Entwicklerin autorisierte deutsche Übersetzung des BESTest zur Verfügung. Diese vorläufigen Ergebnisse bestätigen die Reliabilität und die Validität der Originalversion.

\title{
Schlüsselwörter
}

Balance Evaluation Systems Test · Interkulturelle Übersetzung · Messinstrument · Validität · Reliabilität · Sturzgefahr

Im Gegensatz zu anderen standardisierten Assessments zur Erfassung der Balancefähigkeit trifft der Balance [14] Evaluation Systems Test (BESTest) nicht nur eine Aussage, ob ein Balancedefizit vorliegt, sondern unterstützt Therapeut*innen dabei, das dem Balancedefizit zugrunde liegende System zu identifizieren.

\section{Hintergrund}

Balanceeinschränkungen und Sturzgefahr bei älteren Menschen und Menschen mit neurologischen Erkrankungen werden von Physiotherapeut*innen mit standardisierten Assessments erfasst [24]. Balance-Assessments wie die Berg Balance Scale (BBS) [3, 9], der Tinetti-Test oder der Timedup-go-Test dienen v. a. der Prognose oder der Evaluation [27]. Der BESTest erlaubt 
ebenfalls eine Einschätzung der Sturzgefahr, wurde aber v.a. entwickelt, um Therapeut*innen ein Assessment zur physiotherapeutischen Diagnosestellung an die Hand zu geben [14].

Seine Entwicklerin Fay Horak legte dem BESTest die Systemtheorie von Bernstein zugrunde [4] und beschreibt 6 interagierende Systeme der posturalen Kontrolle: biomechanische Einschränkungen, Stabilitätsgrenzen, antizipatorische Haltungsanpassung, reaktive Haltungsanpassung, sensorische Orientierung und Stabilität im Gang [14].

Der BESTest zeigte in der Testung von älteren Menschen gute bis exzellente Reliabilität und Validität [1, 20]. Es liegen Werte zur Interpretation der Testergebnisse für die Balancetestung älterer Menschen vor (u.a. der Minimal Detectable Change (MDC) [1]).

Eine deutsche Übersetzung der Originalversion war bisher nicht vorhanden. Daher waren die Übersetzung und kulturelle Anpassung des BESTest ins Deutsche primäre Ziele dieser Studie. Das sekundäre Ziel war eine erste Testung der Gütekriterien des BESTest bei Menschen mit Balanceeinschränkungen.

\section{Methode}

Die Genehmigung zur Übersetzung wurde bei der Entwicklerin Fay Horak eingeholt. Ein positives Ethikvotum der Ethikkommission der Universität Koblenz-Landau (Campus Landau) lag vor (Antrag 45). Der Übersetzungsprozess und die kulturelle Anpassung erfolgten in Anlehnung an Beaton [2]. Die folgenden 7 Schritte wurden dabei durchlaufen:

- Schritt 1: Vorwärtsübersetzung durch

2 voneinander unabhängige Personen

(eine mit dem BESTest vertraute und in den USA promovierte Physiotherapeutin; eine professionelle Übersetzerin ohne medizinisch-therapeutischen Hintergrund),

- Schritt 2: Synthese der beiden Übersetzungen zu einer vorläufigen deutschen Version; Kennzeichnung diskrepanter Formulierungen für die Expert*innenkonferenz,

- Schritt 3: Rückübersetzung der vorläufigen Version durch 2 voneinander unabhängige Muttersprachler*innen (eine Physiotherapeutin, mit dem BESTest nicht vertraut; eine Person ohne medizinische Kenntnisse); Vergleich der Rückübersetzungen mit der Originalversion; Protokollierung von Diskrepanzen, Anmerkungen und Unklarheiten für die Expert*innenkonferenz,

- Schritt 4: Durchführung der Expert*innenkonferenz mit 7 Teilnehmenden (alle an der Übersetzung Beteiligten, ein Patient*innenvertreter, ein Psychologe mit methodischer Expertise und die Projektkoordinator*innen); Besprechung und Lösung aller Anmerkungen und Unklarheiten, Erstellen der vorläufigen Version,

- Schritt 5: Testung der vorläufigen Version im Feld,

- Schritt 6: Autorisierung der finalen Version durch die Entwicklerin des BESTest,

- Schritt 7: erste Überprüfung der Gütekriterien der übersetzten Version bei Menschen mit Balancedefiziten.

\section{Verständlichkeit}

\section{Teilnehmende}

Sieben Physiotherapeutinnen und ein Physiotherapeut aus Deutschland, Österreich und der Schweiz überprüften die Verständlichkeit. Die Therapeut*innen arbeiteten in 2 neurologischen Einrichtungen, in einer orthopädischen Praxis sowie 2 psychiatrischen Kliniken. Sieben Physiotherapeut*innen hatten über 10 Jahre Berufserfahrung, eine Physiotherapeut*in zwischen 2 und 5 Jahren; vier Teilnehmende hatten einen akademischen Abschluss.

\section{Instrumente}

Mit einem Fragebogen wurden für jedes Item die Verständlichkeit der "Instruktionen für die Therapeut*innen", "Instruktionen für Patient*innen" sowie die Verständlichkeit des Bewertungsschemas geprüft. Die Antwortmöglichkeiten waren „Ja“ oder „Nein“. Falls „Nein“ angekreuzt wurde, wurde um Begründung gebeten.

\section{Ablauf}

Fünf Therapeut*innen führten den BESTest mit Patient*innen durch und füllten im
Anschluss daran den Fragebogen aus. Drei weitere Therapeut*innen überprüften die Textverständlichkeit ohne $\mathrm{Pa}$ tient*innenkontakt.

\section{Datenauswertung}

Die offenen Antworten wurden gesammelt. Folgende Kategorien wurden vorab gebildet:

1. Orthographie,

2. semantisches Verständnis,

3. pragmatisches Verständnis.

Während der Auswertung wurde eine zusätzliche Kategorie eingeführt:

4. Originalquelle BESTest/Testkonstruktion.

\section{Gütekriterien}

Teilnehmende

Die Ad-hoc-Stichprobe wurde über eine Selbsthilfegruppe für Menschen mit idiopathischem Parkinson-Syndrom, eine physiotherapeutische Praxis sowie über ein ambulantes Rehazentrum rekrutiert. Einschlusskriterium war ein subjektiv empfundenes oder objektiv festgestelltes Balancedefizit. Ausgeschlossen wurden Personen mit kognitiven Einschränkungen.

\section{Instrumente zur Überprüfung der} Validität

Den 6 interagierenden Balancesystemen des BESTest werden insgesamt 36 Items zugeordnet. Jedes Item wird auf einer 4-stufigen Skala von 0 bis 3 Punkten bewertet, wobei eine höhere Punktzahl eine bessere Balancefähigkeit bedeutet. Die maximale Punktzahl beträgt 108 Punkte, wobei sich diese mit Punktwerten zwischen 15 und 21 Punkten auf die einzelnen Systeme verteilt und die Ergebnisse sowohl für die einzelnen Systeme als auch für den Gesamttest als Prozentwerte angegeben werden können [14]. Um die Kriteriumsvalidität des BESTest zu überprüfen, wurde ein beobachtungsbasiertes Instrument, die BBS, genutzt. Zur Überprüfung der Konstruktvalidität wurde ein Selbstauskunftsinstrument zur Einschätzung der Sturzangst, die deutsche Activities-Specific Balance Con- 


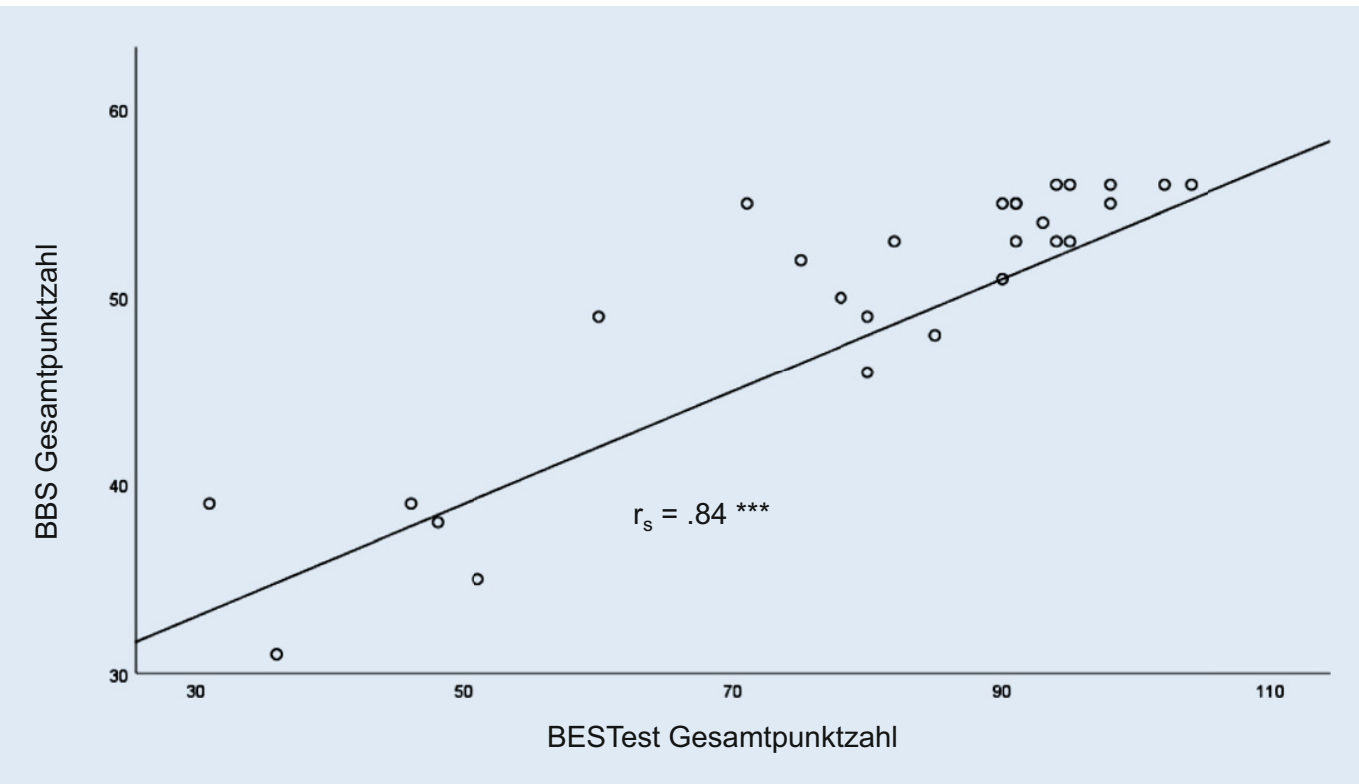

Abb. $1 \varangle$ Korrelation des BESTest mit der Berg Balance Scale (BBS, $n=27$, $\left.{ }^{* * *} p<0,001\right)$

fidence Scale (ABC-D Scale) als konstruktnahes Instrument gebraucht [26].

\section{Ablauf}

Vor der Durchführung des BESTest bzw. der BBS wurden demografische Daten, inklusive Sturzhistorie, erhoben, und die Teilnehmenden wurden gebeten, die ABC-D Scale auszufüllen. Die Reihenfolge der Testdurchführung war ausbalanciert, um Reihenfolgeeffekte zu vermeiden.

\section{Datenanalyse}

Obwohl die Entwicklerin ein Ordinalskalenniveau beschreibt [14], wird in der internationalen Literatur mit einem intervallskalierten Niveau gerechnet [1, 19, 23]. Daher wurde ein Intervallskalenniveau des BESTest angenommen. Für alle Assessments wurden Mittelwert, Standardabweichung sowie der minimale und maximale Wert berechnet. Zusätzlich wird, um mögliche Deckeneffekte aufzuzeigen, der Prozentsatz der Teilnehmenden, die den maximalen Wert erzielten, berichtet. Die Normalverteilungsannahme wurde mithilfe des Shapiro-Wilk-Tests überprüft. Die interne Konsistenz des BESTest wurde mit Cronbachs a berechnet. Basierend auf internationalen Studien $[5,7]$ wurde ein $a>0,7$ erwartet. Zur besseren Interpretation der Ergebnisse wird das a für ordinale Daten berichtet [12]. Die Interpretation der Werte des ordinalen a erfolgt synonym zu den Cronbachs-a-Werten.

Zur Bestimmung der Kriteriumsvalidität wurden die Werte des BESTest mit denen der BBS mithilfe von Spearmans $\rho$ korreliert. Die Konstruktvalidität wurde mit der konstruktnahen $A B C-D S c a l e$ ebenfalls mittels Spearmans $\rho$ berechnet. Es wird erwartet, dass eine hohe Korrelation vorliegt [14]. Außerdem wurde mithilfe der Methode der bekannten Gruppen ermittelt, ob der BESTest zwischen gestürzten und nichtgestürzten Personen unterscheiden kann. Dabei wurde angenommen, dass gestürzte Personen einen geringeren BESTestWert aufweisen als nichtgestürzte Personen [22]. Aufgrund der Voraussetzungsverletzung der Varianzhomogenität der Gruppen wurde der Mann-Whitney-U-Test verwendet.

Zur Prüfung der Differenzierungsfähigkeit des BESTest wurden exemplarisch Ergebnisse von 4 Personen mit unterschiedlichen Grunderkrankungen/ Einschränkungen, die im Gesamttest bis auf $1 \%$ das gleiche Ergebnis erzielten, dargestellt [14].

\section{Ergebnisse}

\section{Verständlichkeit}

Insgesamt lagen 75 Rückmeldungen zur Verständlichkeit der "Instruktionen für die Therapeut*innen“, der „Instruktionen für die Patient*innen“ und zur Verständlichkeit der Bewertung der Items vor.

Nach Absprache mit der Entwicklerin wurden folgende Änderungen vorgenommen:

1. Zusammenführung der "Instruktion für die Therapeut*in" von S. 1 mit der Instruktion zur Interrater-Reliabilität auf S. 2,

2. Hinzufügen des Links für die Trainingsvideos auf S. 1,

3. Zusammenführung der "Instruktionen für Therapeut ${ }^{*}$ innen und Patient*innen" mit dem Bewertungsschema,

4. Angleichung des Bewertungstexts bei Item 16 und Item 17,

5. Übersetzung des Begriffs "tempur pad" mit dem Begriff „Airex Balance Pad" ins Deutsche,

6. Übersetzung von "4-12 feet" mit "1-4 m",

7. Übersetzung von „assistive device" als "Stand- und Gangunterstützung durch einen Stock oder Gehbock",

8. Übersetzung von „physical assistance“ als „manuelle Unterstützung durch den*die Therapeut ${ }^{*}$ in oder ein Halten an der Bank",

9. die Phrase "requires touch assist" in die Bewertung des Item 20 inkludiert sowohl die Hilfe eines Stocks als auch manuelle Unterstützung durch den*die Therapeut*innen. 


\begin{tabular}{|l|l|}
\hline Tab. 1 Teilnehmenden-Charakteristika \\
\hline Variable & $\begin{array}{l}\text { Anzahl der Teil- } \\
\text { nehmenden } \\
(n=27)\end{array}$ \\
\hline Geschlecht (m/w) & $10 / 17$ \\
\hline $\begin{array}{l}\text { Alter MW ( } \pm \text { ); Min- } \\
\text { Max }\end{array}$ & $\begin{array}{l}56,70 \text { ( } \pm 16,25) ; \\
24-80\end{array}$ \\
\hline Grunderkrankung/Symptom (n) \\
\hline $\begin{array}{l}\text { Idiopathisches Parkin- } \\
\text { son-Syndrom }\end{array}$ & 6 \\
\hline $\begin{array}{l}\text { "Upper motor neuron } \\
\text { syndrome" }\end{array}$ & 8 \\
\hline $\begin{array}{l}\text { Muskuloskeletal } \\
\text { Erkrankung von } \\
\text { UEX+WS }\end{array}$ & 6 \\
\hline $\begin{array}{l}\text { Rückenmark- } \\
\text { schädigungen }\end{array}$ & 2 \\
\hline $\begin{array}{l}\text { Neuromuskuläre Er- } \\
\text { krankungen }\end{array}$ & 2 \\
\hline Subjektive Instabilität & 3 \\
\hline Sturz (ja/nein) & $15 / 12$ \\
\hline $\begin{array}{l}m \text { männlich, } w \text { weiblich, MW Mittelwert, } \\
\text { SD Standardabweichung, Min minimaler } \\
\text { Wert, Max maximaler Wert, UEX untere Extre- } \\
\text { mität, WS Wirbelsäule }\end{array}$ \\
\hline
\end{tabular}

\section{Gütekriterien}

Die demografischen Daten der 27 Teilnehmenden beschreibt $\boldsymbol{0}$ Tab. 1. Fünfzehn Teilnehmende berichteten über einen Sturz in der Vergangenheit. Die Ergebnisse in allen 3 Balance-Assessments zeigt - Tab. 2. Dabei erzielten die Teilnehmenden im Gesamt-BESTest einen Mittelwert von 80 Punkten $( \pm 20,81)$. In der BBS lag der Mittelwert bei $50( \pm 7,22)$ von maximal 56 Punkten. Der durchschnittliche Prozentwert der ABC-D Scale lag bei $73 \%( \pm 20,77)$. Keine Person erreichte im BESTest die maximale Punktzahl; im Vergleich erzielten $5 \%$ der Teilnehmenden in der BBS und 3,7\% in der ABC-D Scale die maximale Punktzahl.

Cronbachs a zur Bestimmung der internen Konsistenz betrug 0,95 für die Gesamtskala. Das a für ordinale Daten betrug ebenfalls 0,95. Die Ergebnisse der Kriteriumsvalidität zeigt • Abb. 1, wobei die Werte des BESTest mit denen der BBS hoch korrelieren $(\rho=0,84 ; p=<0,001)$.

Auch die Konstruktvalidität wurde bestätigt, wobei die Werte der ABC-D Scale mit denen des BESTest hoch korrelieren $(\rho=0,61 ; p=<0,001 ; \square$ Abb. 2$)$. Ebenso erzielten die nichtgestürzten Teilnehmen-

\begin{tabular}{|l|l|l|l|l|}
\hline Tab. 2 Ergebnisse, BESTest, BBS, ABC-D Scale \\
\hline Assessment & $\begin{array}{l}\text { Mögliche } \\
\text { Spanne }\end{array}$ & MW (SD) & $\begin{array}{l}\text { Mini- } \\
\text { mum }\end{array}$ & $\begin{array}{l}\text { Maxi- } \\
\text { mum }\end{array}$ \\
\hline BESTest & $0-108$ & $79,59(20,81)$ & 31 & 104 \\
\hline Gesamt BESTest & $0-15$ & $9,88(2,79)$ & 5 & 14 \\
\hline I: Biomechanische Einschränkungen & $0-21$ & $17,55(2,29)$ & 13 & 21 \\
\hline II: Stabilitätslimits/Vertikalität & $0-18$ & $12,96(3,90)$ & 5 & 18 \\
\hline $\begin{array}{l}\text { III: Bewegungsübergänge, antizipato- } \\
\text { risch }\end{array}$ & $0-18$ & $11,51(5,97)$ & 0 & 18 \\
\hline IV: reaktive Haltungsanpassung & $0-15$ & $12,59(3,14)$ & 4 & 15 \\
\hline V: sensorische Orientierung & $0-21$ & $15,07(5,77)$ & 2 & 21 \\
\hline VI: Stabilität im Gehen & $0-56$ & $49,93(7,22)$ & 31 & 56 \\
\hline $\begin{array}{l}\text { BBS } \\
\text { ABC-D Scale }\end{array}$ & $0-100 \%$ & $73,35 \%(20,77)$ & $13 \%$ & $100 \%$ \\
\hline $\begin{array}{l}\text { MW Mittelwert, SD Standardabweichung, \% Prozent, BESTest Balance Evaluation Systems Test, } \\
\text { BBS Berg Balance Scale, ABC-D Scale deutsche Activities-Specific Balance Confidence Scale, I-V Sys- } \\
\text { tem I bis System VI }\end{array}$ \\
\hline
\end{tabular}

den durchschnittlich einen höheren Wert im BESTest $(87,71( \pm 14,21)$ als gestürzte Personen $(73,40( \pm 23,42)$; allerdings verfehlte dieser Unterschied die Signifikanzschwelle $(U=51,00, Z=-1,905333$, $p=0,057)$ ).

Exemplarisch zeigt • Abb. 3 die Ergebnisse von 4 ausgewählten Teilnehmenden. Während sie vergleichbare Gesamtprozentwerte erreichen, weisen sie jedoch gleichzeitig unterschiedliche Ergebnisse in den einzelnen Systemen auf. Zum Beispiel erreicht die Person mit idiopathischem Parkinson-Syndrom im System 1 den niedrigsten Wert, die Person mit dem „upper motor neuron syndrome“ hingegen im System 5.

\section{Diskussion}

Mit dieser Arbeit steht eine von der Entwicklerin autorisierte und nach internationalen Leitlinien [2] angefertigte deutsche Übersetzung des BESTest zur Verfügung. Erste Ergebnisse zu den Gütekriterien wurden anhand einer geriatrischen und neurologischen Stichprobe ermittelt.

Die Gesamtskala des deutschen BESTest zeigte für intervallskalierte und ordinale Daten exzellente Werte für die interne Konsistenz. Dies deckt sich mit der internationalen Literatur [5, 7, 25]. Die Ergebnisse aus der Bestimmung der konkurrenten Validität des BESTest mit der BBS und ABC-D Scale bestätigen dessen Kriteriums- und Konstruktvalidität. Wie bereits in der Literatur [1, 6, 15-18, 20, 22, 25] berichtet, konnte auch für die deutsche Version
- über unterschiedliche Diagnosen hinweg - ein hoher Zusammenhang mit der $B B S$ und der ABC-D Scale gefunden werden. Die Bestimmung der Konstruktvalidität anhand bekannter Gruppen [8] verfehlte die Signifikanz knapp $(p=0,057)$. Eine vergleichbare Studie fand ebenfalls einen Trend zur Signifikanz [22].

Der BESTest zeichnet sich darüber hinaus - im Vergleich zur BBS - durch geringere Deckeneffekte aus. Während 5\% der Stichprobe in der BBS die maximale Punktzahl erreichten, erreichte diese keine Person im BESTest. Dies deckt sich mit den Ergebnissen zur englischsprachigen Originalversion [20]. Auch Gordt fand höhere Deckeneffekte in der BBS im Vergleich zu der von ihr übersetzten Community Balance and Mobility Scale [13].

\section{Praktische Implikationen}

Eine Übersichtsarbeit, die 66 BalanceAssessments einschloss, zeigte, dass der BESTest als einziges Assessment unterschiedliche, für die Balance verantwortliche Systeme untersucht [28]. Eine solche Untersuchung ermöglicht die Identifikation defizitärer Systeme bei vorliegenden Balanceproblemen, die in der Therapie spezifisch adressiert werden können [14]. Die exemplarische Darstellung in • Abb. 3 bestätigt diese Differenzierungsmöglichkeit auch für die deutsche Version. Ein zusätzlicher Mehrwert des BESTest ist die detaillierte Testung der reaktiven Balance, die mit 5 Items standardisiert untersucht 


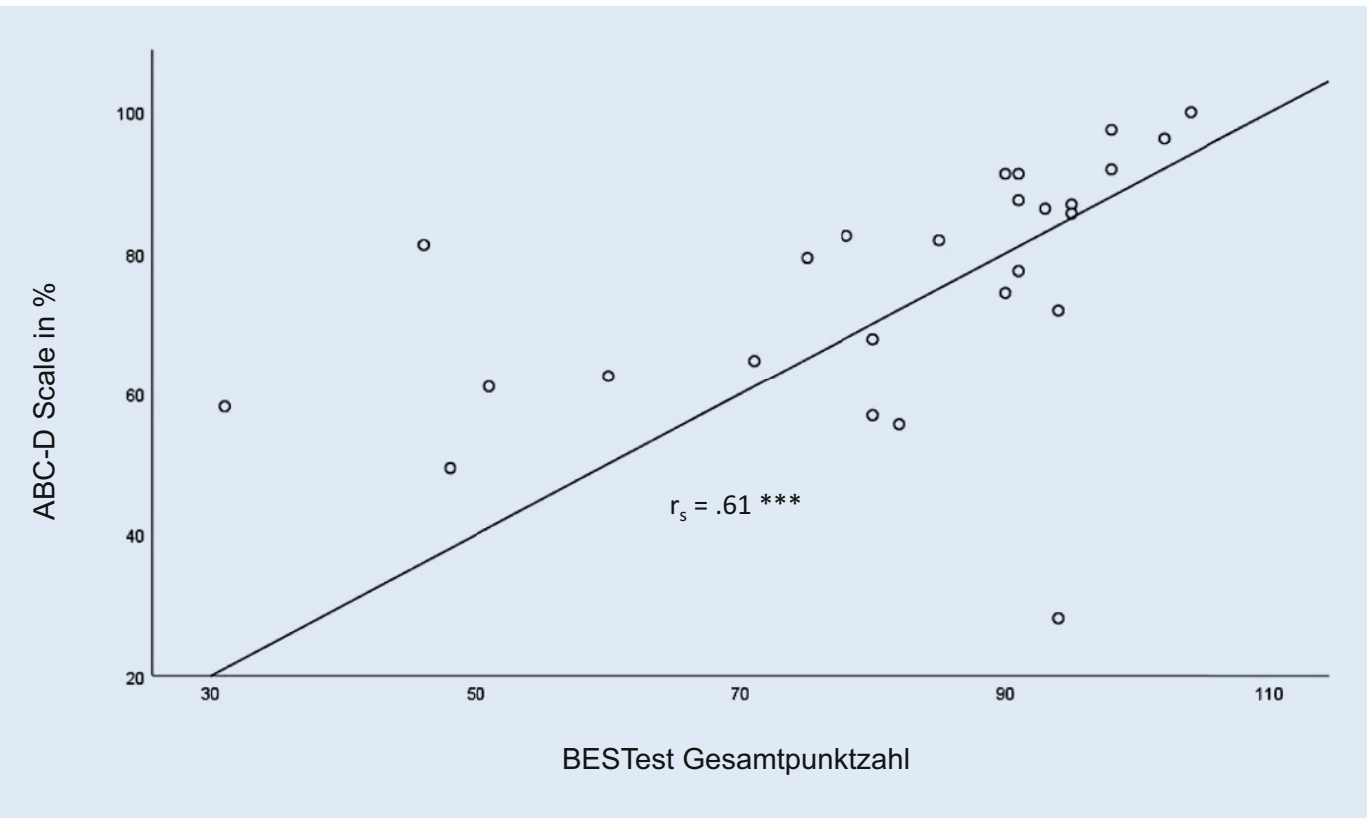

Abb. $2<$ Korrelation des BESTest mit der ActivitiesSpecific Balance Confidence Scale (ABC-D Scale, $n=27 ; * * * ; p<0,001)$

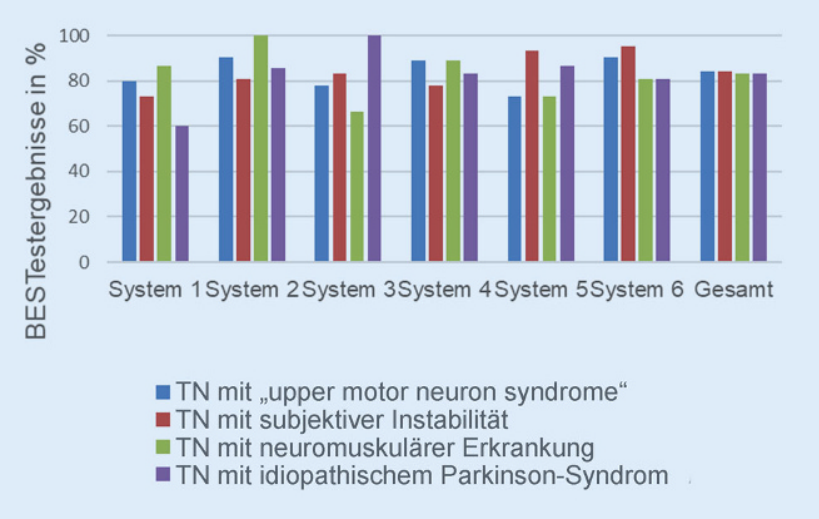

wird. In den gängigen Assessments (z. B. BBS) wird die reaktive Balance nicht oder nur sehr allgemein und ohne klare Standardisierung (z. B. Tinetti-Test) evaluiert.

Ein Nachteil des BESTest ist dessen Durchführungsdauer (ca. $30 \mathrm{~min}$ ). In diesem Zusammenhang wurden 2 kürzere Versionen entwickelt: der Mini-BESTest [11] und der Brief-BESTest [23]. Bei älteren Menschen wird der Einsatz des Brief-BESTest empfohlen [20, 21, 29], da dieser weniger Zeit und Material in Anspruch nimmt als der Mini-BESTest und die Originalversion des BESTest [10].

\section{Stärken und Schwächen der Arbeit}

Die Aussagekraft der Studie ist durch die geringe Stichprobengröße limitiert. Allerdings sind unsere ersten Ergebnisse mit denen aus internationalen Untersuchungen vergleichbar [5, 7, 14, 20, 25].

An der Verständlichkeitstestung nahmen 8 Therapeut*innen aus 3 deutschsprachigen Ländern sowie akademisierte und nichtakademisierte Therapeut*innen teil. Dies erleichtert die Anwendbarkeit im deutschsprachigen Berufsfeld.

\section{Fazit für die Praxis}

- Aufgrund erster Bestätigung der Gütekriterien empfiehlt sich der Einsatz in der Praxis und Forschung.

- Der deutschsprachige BESTest ist http:// www.bestest.us/files/2115/3299/3497/ German_BESTest_v2.pdf online abrufbar.

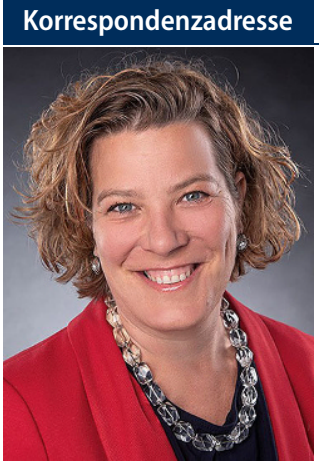

Maren Haselwander, Dipl.-Psych. PT Fakultät für Therapiewissenschaften, SRH Hochschule Heidelberg Maria-Probst-Str. 3, 69123 Heidelberg Deutschland maren.haselwander@srh.de

Danksagung. Unser Dank gilt Frau PD Dr. Dlugosch, allen am Übersetzungsprozess Beteiligten sowie den Testteilnehmenden.

Förderung. Die Studie wurde von der Förderstiftung der SRH Holding unterstützt.

Funding. Open Access funding enabled and organized by Projekt DEAL.

\section{Einhaltung ethischer Richtlinien}

Interessenkonflikt. M. Haselwander, Y. Henes, M. Weisbrod und G. Diermayr geben an, dass kein Interessenkonflikt besteht. 
Die Studie wurde mit Zustimmung der Ethikkommission der Universität Koblenz-Landau (Campus Landau - Antrag 45) gemäß der Deklaration von Helsinki durchgeführt. Von allen Teilnehmenden liegt eine Einverständniserklärung vor.

Open Access. Dieser Artikel wird unter der Creative Commons Namensnennung 4.0 International Lizenz veröffentlicht, welche die Nutzung, Vervielfältigung, Bearbeitung, Verbreitung und Wiedergabe in jeglichem Medium und Format erlaubt, sofern Sie den/die ursprünglichen Autor(en) und die Quelle ordnungsgemäß nennen, einen Link zur Creative Commons Lizenz beifügen und angeben, ob Änderungen vorgenommen wurden.

Die in diesem Artikel enthaltenen Bilder und sonstiges Drittmaterial unterliegen ebenfalls der genannten Creative Commons Lizenz, sofern sich aus der Abbildungslegende nichts anderes ergibt. Sofern das betreffende Material nicht unter der genannten Creative Commons Lizenz steht und die betreffende Handlung nicht nach gesetzlichen Vorschriften erlaubt ist, ist für die oben aufgeführten Weiterverwendungen des $\mathrm{Ma}$ terials die Einwilligung des jeweiligen Rechteinhabers einzuholen.

Weitere Details zur Lizenz entnehmen Sie bitte der Lizenzinformation auf http://creativecommons.org/ licenses/by/4.0/deed.de.

\section{Literatur}

1. Anson E, Thompson E, Ma L et al (2019) Reliability and fall risk detection for the BEStest and miniBEStest in older adults. J Geriatr Phys Ther 42(42):81-85

2. Beaton $D E$, Bombardier $C$, Guillemin $F$ et al (2000) Guidelines for the process of crosscultural adaptation of self-report measures. Spine 25(24):3186-3191

3. Berg KO, Wood-Dauphinee SL, Williams Jl et al (1992) Measuring balance in the elderly. Can J Public Health 83(Suppl 2):7-11

4. Bernstein NA (1988) Bewegungsphysiologie, 2. Aufl. J.A. Barth, Leipzig

5. Bravini E, Nardone A, Godi M et al (2016) Does the Brief-BEStest meet classical test theory and Rasch analysis requirements for balance assessment in people with neurological disorders? Phys Ther 96(10):1610-1619

6. Chiu AYY, Pang MYC (2017) Assessment of psychometric properties of various balance assessment tools in persons with cervical spondylotic myelopathy. J Orthop Sports Phys Ther 47(9):673-682

7. Dominguez-Olivan P, Gasch-Gallen A, AguasGarcia E et al (2020) Validity and reliability testing of the Spanish version of the BESTest and miniBESTest in healthy community-dwelling elderly. BMC Geriatr 20(1):444

8. Döring N, Bortz J (2016) Forschungsmethoden und Evaluation in den Sozial- und Humanwissenschaften, 5. Aufl. Springer, Berlin, Heidelberg

9. Downs S(2015) The Berg balancescale.JPhysiother 61(1):46

10. Duncan RP, Leddy AL, Cavanaugh JT et al (2013) Comparative utility of the BESTest, mini-BESTest, and brief-BESTest for predicting falls in individuals with Parkinson disease. Phys Ther 93(4):542-550

11. Franchignoni F, Horak F, Godi $M$ et al (2010) Using psychometric techniques to improve the balance evaluation systems test. J Rehabil Med 42(4):323-331

\section{Balance Evaluation Systems Test: German translation, cultural adaptation and preliminary results on psychometric properties}

Background: The Balance Evaluation Systems Test (BESTest) evaluates balance ability and identifies affected systems associated with balance deficits.

Objective: The primary objective was the translation and cultural adaptation of the BESTest into German. Secondary objective was the preliminary evaluation of psychometric properties of the BESTest.

Method: In accordance with international guidelines, the BESTest was translated and adapted by a 7-step process. To test the psychometric properties, persons with subjective or objective balance deficits were included. Internal consistency was evaluated by means of Cronbach's alpha. The criterion validity was tested by measuring the correlation with the Berg Balance Scale (BBS). Construct validity was examined using the Activities-Specific Balance Confidence ( $A B C-D)$ scale and the known groups methods (fallers versus non-fallers).

Results: A total of 27 subjects were included in the testing of the psychometric properties. Cronbach's alpha was 0.95 for the total scale. The correlation of BESTest scores with those of the BBS revealed a Spearmen's $\rho$ of $0.84(p<0.001)$ and 0.61 $(p<0.001)$ with those of the ABC-D scale. Fallers and non-fallers showed a trend towards different BESTest scores ( $p=0.057)$.

Discussion: This paper provides a German translation of the BESTest authorized by the developer. Our preliminary results confirm reliability and validity.

\section{Keywords}

Balance Evaluation Systems Test · Cross-cultural translation · Outcome measures · Validity . Reliability · Fall risk

12. Gadermann AM, Guhn M,Zumbo BD (2012) Estimating ordinal reliability for Likert-type and ordinal item response data: A conceptual, empirical, and practical guide. https://www.researchgate.net/ publication/236605201_Estimating_ordinal_ reliability_for_Likert-type_and_ordinal_item_ response_data_A_conceptual_empirical_and_ practical_guide.Zugegriffen: 1.Juni 2021

13. Gordt K, Mikolaizak AS, Nerz C et al (2019) Deutschsprachige Version derCommunityBalance and Mobility Scale : Übersetzung und Evaluation der Gütekriterien. Z Gerontol Geriat 52(1):28-36

14. Horak FB, Wrisley DM, Frank J (2009) The Balance Evaluation Systems Test (BESTest) to differentiate balance deficits. Phys Ther 89(5):484-498

15. Huang MH, Miller K, Smith Ket al (2016) Reliability, validity, and minimal detectable change of balance evaluation systems test and its short versions in older cancer survivors. J Geriatr Phys Ther 39(2):58-63

16. Jacobs JV, Kasser SL (2012) Balance impairment in people with multiple sclerosis. Gait Posture 36(3):414-418

17. Jácome C, Cruz J, Oliveira A et al (2016) Validity, reliability, and ability to identify fall status of the berg balance scale, BEStest, mini-BEStest, and brief-BEStest in patients with COPD. Phys Ther 96(11):1807-1815

18. Leddy AL, Crowner BE, Earhart GM (2011) Functional gaitassessmentand balance evaluation system test. Phys Ther 91(1):102-113

19. Maia AC, Rodrigues-de-Paula F, Magalhães LC et al (2013) Cross-cultural adaptation and analysis of the psychometric properties of the balance evaluation systems test and MiniBEStest in the elderly and individuals with parkinson's disease. Braz JPhys Ther 17(3):195-217
20. Marques A, Almeida S, Carvalho J et al (2016) Reliability, validity, and ability to identify fall status of the balance evaluation systems test, mini-balance evaluation systems test, and briefbalance evaluation systems test in older people living in the community. Arch Phys Med Rehabil 97(12):2166-2173.e1

21. McLay R, Kirkwood RN, Kuspinar A et al (2020) Validity of balance and mobility screening tests for assessing fall risk in COPD. Chron Respir Dis 17:1479973120922538

22. O'Hoski S, Sibley KM, Brooks D et al (2015) Construct validity of the BESTest, mini-BESTest and briefBESTest in adults aged 50 years and older. Gait Posture 42(3):301-305

23. Padgett PK, Jacobs JV, Kasser SL (2012) Is the BESTest at its best? A suggested brief version based on interrater reliability, validity, internal consistency, and theoretical construct. Phys Ther 92(9):1197-1207

24. Potter K, Fulk GD, Salem Y et al (2011) Outcome measuresinneurological physical therapypractice: part I. Making sound decisions. J Neurol Phys Ther 35(2):57-64

25. Potter K, Anderberg L, Anderson D et al (2017) Reliability, validity, and responsiveness of the balance evaluation systems test (BEStest) in individuals with multiple sclerosis. Physiotherapy 104(1):142-148

26. Schott N (2008) Deutsche Adaptation der „Activities-Specific Balance Confidence (ABC) Scale" zur Erfassung dersturzassoziierten Selbstwirksamkeit. ZGerontol Geriat 41(6):475-485

27. Sibley KM, Straus SE, Inness EL et al (2013) Clinical balance assessment: perceptions of commonlyused standardized measures and current practices among physiotherapists in Ontario, Canada. Implement Sci 8:33 
28. Sibley KM, Beauchamp MK, van Ooteghem K et al (2015) Using the systems framework for postural control to analyze the components of balance evaluated in standardized balance measures:a scoping review. Arch Phys Med Rehabil 96(1):122-132.e29

29. Viveiro LAP, Gomes GCV, Bacha JMR et al (2019) Reliability, validity, and ability to identity fall status of the berg balance scale, balance evaluation systems test (BEStest), mini-BEStest, and briefBEStest in older adults who live in nursing homes. J Geriatr Phys Ther 42(4):E45-E54
Hermann J. Berberich, Désirée-Louise Dräger

\section{Psychosoziale Uroonkologie}

Berlin, Heidelberg: Springer 2022, 1, 220 S., (ISBN: 978-3-662-65091-2 ), 49,99 EUR

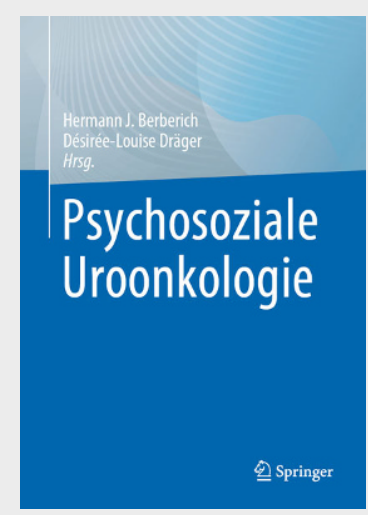

Ca. ein Viertel aller Tumorerkrankungen sind dem urologischen Fachge-biet zuzurechnen. Die Krebsdiagnose selbst stellt für die Betroffenen und ihre Angehörigen häufig die größte Herausforderung ihres bisherigen Lebens dar. Individuelle Vorgeschichte und persönliche Perspektiven bestimmen Art und Ausmaß der mit der Erkrankung verbundenen psychischen Belastungen.

Viele Aspekte der psychoonkologischen/psychosozialen Betreuung folgen interdisziplinär übergreifenden Regeln, die in der Fachliteratur fundiert verankert sind. Darüber hinaus gibt es aber zahlreiche Spezifika der urologisch orientierten psychosozialen Betreuung. Beispielhaft betrifft dies im urologisch-onkologischen Klientel den hohen Anteil an Männern im höheren Lebensalter mit den in dieser Gruppierung weit verbreiteten besonderen Konfliktlösungsstrategien und daraus abzuleitenden spezifischen Betreuungs-Erfordernissen. Zu den Besonderheiten der Urologie schließt das vorgestellte Lehrbuch die bislang als deutlich wahrgenommene Lücke in der psychoonkologischen Literatur auf Fachbuch-Ebene.

Den Herausgebern und neun weiteren Autoren ist es hervorragend gelungen, die psychosoziale Uroonkologie in allen relevanten Facetten darzustellen. Die komplexe Thematik wird auf 220 Seiten sehr übersichtlich unter Verwendung verständlicher Schemata und informativer Abbildungen kompetent, kompakt und interessant vermittelt - Lehrbuch und Nachschlagewerk gleichermaßen.

\section{Praxisnahe Fallvignetten}

Sehr praxisnah spiegeln die verwendeten Fallvignetten das psychosoziale Arbeiten abgestellt auf die bei den verschiedenen urologischen Tumorentitäten durchaus differenten Besonderheiten und Herausforderungen der psychosozialen Betreuung.

Das Lehrbuch „Psychosoziale Uroonkologie“ bietet mit seiner aktuellen umfassenden Darstellung auch allgemeiner fachübergreifender psychoonkologischer Aspekte und zugehöriger Versorgungskonzepte und -angebote prinzipiell allen psychoonkologisch Interessierten wertvolle Informationen.

Die gute Übersichtlichkeit und Struktur der Darstellung machen es für jeden Urologen, selbst wenn er nur gelegentlich mit psychoonkologischen Fragestellungen konfrontiert sein sollte, zu einem wertvollen Nachschlagewerk.

Denen, die urologische Tumorpatienten psychosozial umfassend betreuen, sollte das Werk ein zentrales Instrumentarium ihrer herausfordernden Arbeit sein. 